\title{
CONCENTRACIÓN DE MACRONUTRIMENTOS Y MICRONUTRIMENTOS EN GRANOS DE CAFÉ (Coffea sp.) DE DIFERENTES ORIGENES
}

\author{
CONCENTRATION OF MACRONUTRIENTS AND MICRONUTRIENTS \\ IN COFFEE BEANS (Coffea sp.) OF DIFFERENT ORIGINS
}

\begin{abstract}
Trejo-Téllez, L.I. ; Gómez-Merino, F.C. ${ }^{1 *}$; Morales-Ramos, V. ${ }^{1}$; Marín-Garza, T. ${ }^{2}$; Castañeda-Castro, O. ${ }^{3}$; Pastelin-Solano, M.C. ${ }^{3}$
${ }^{1}$ Colegio de Postgraduados Campus Montecillo. Carretera México-Texcoco km 36.5. Montecillo, Texcoco, Estado de México. C. P. 56230. ²Universidad Veracruzana. Facultad de Ciencias Biológicas y Agropecuarias Zona Córdoba-Orizaba. Carretera Peñuela-Amatlán km 1. Peñuela, Amatlán de los Reyes, Veracruz. C. P. 94945. ${ }^{3}$ Universidad Veracruzana. Facultad de Ciencias Químicas Zona Córdoba-Orizaba. Calle Oriente 6 No. 1009, Col. Rafael Alvarado, Orizaba, Veracruz. C. P. 94340.
\end{abstract}

*Autor de correspondencia: fernandg@ecolpos.mx

\section{RESUMEN}

La constitución nutrimental de las plantas, y en específicos de sus semillas o granos, depende de factores como la variedad el clima, el manejo agronómico y la composición mineral del suelo donde se cultivan. A pesar de ser un país productor de café, en México existen pocos estudios comparativos sobre el efecto del origen de los granos en la calidad del grano de café. En esta investigación se determinó la concentración de macronutrimentos ( $N, P, K, C a, M g$ y $S$ ) y micronutrimentos ( $B$, $\mathrm{Cu}, \mathrm{Fe}, \mathrm{Mn}$, Ni y Zn) en granos de café de la variedad Robusta (Coffea canephora) provenientes de Méxixo, Brasil y Vietman, por espectrofotometría de emisión atómica de inducción por plasma acoplado (ICP-IES), con excepción del N, para el cual se empleó el método semi-micro Kjeldahl. Los resultados obtenidos demuestran, que con excepción del N, existe una variación en las concentraciones de los elementos minerales entre los granos los tres distintos países. Las muestras de México presentaron valores por encima del 50\% en P, Ca, K y Mg; y más del 100\% en Fe, Mn y Zn con respecto a los granos de café provenientes de Brasil y Vietnam.

Palabras clave: Coffea canephora, café, paises productores, calidad, nutrimentos.

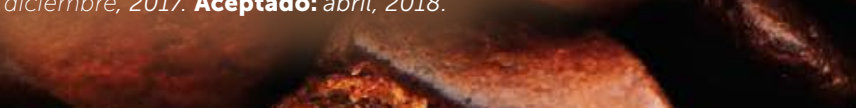

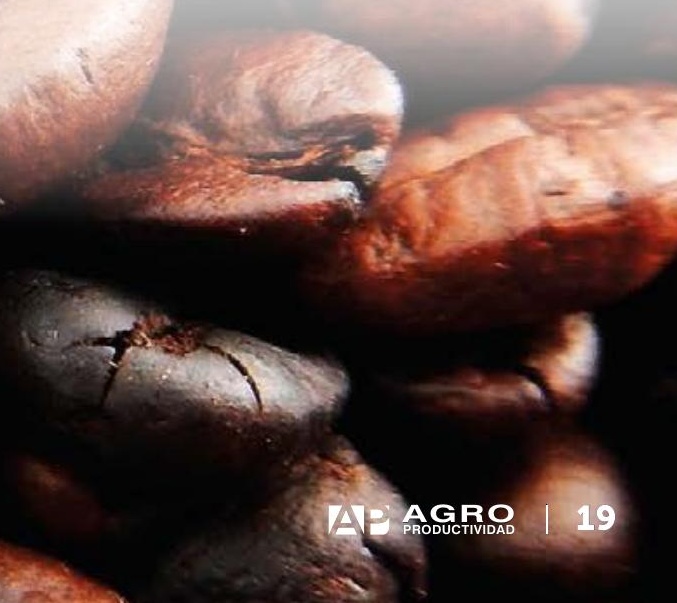




\begin{abstract}
The nutritional constitution of plants, and specifically of their seeds or grains, depends on factors such as the variety, the climate, the agronomic management and the mineral composition of the soil where they are cultivated. Although Mexico is a coffee producing country, there are few comparative studies exploring the effect of the place where grains are grown on the quality of coffee beans. In this study, the concentration of macronutrients ( $N, P, K, C a, M g$ and S) and micronutrients ( $B, C u, F e, M n$, $\mathrm{Ni}$ and $\mathrm{Zn}$ ) in coffee beans of the Robusta variety (Coffea canephora), from three different countries, Mexico, Brazil, and Vietnam, was determined by inductively coupled plasma atomic emission spectroscopy (ICP-AES), with the exception of $\mathrm{N}$, for which the semi-micro Kjeldahl method was used. The results obtained show that, with the exception of $N$, there is a variation in the concentrations of mineral elements among the grains of the regions studied. Samples from Mexico presented values above 50\% in $\mathrm{P}, \mathrm{Ca}, \mathrm{K}$ and $\mathrm{Mg}$; and more than $100 \%$ in $\mathrm{Fe}, \mathrm{Mn}$ and $\mathrm{Zn}$ with respect to coffee beans from Brazil and Vietnam.
\end{abstract}

Keywords: Coffea canephora, coffee, producing countries, quality, nutrients.

\section{INTRODUCCIÓN}

El Oran@ Oe Café se obtiene de la fruta de arbusto de hoja perenne perteneciente al género Coffea de la familia Rubiaceae. Aunque existen varias especies, solamente dos han cobrado importancia económica mundial: Coffea arabica, adaptada a regiones templadas y altitudes más elevadas, de la cual se obtiene aproximadamente el 90\% de la producción total, y Coffea canephora, que es más rústica y mejor adaptada a regiones de baja altitud y altas temperaturas, contribuyendo con 9\% (Kemsley et al., 1995; Sacco et al., 2011; Toledo et al., 2016). Los granos de café de las variedades de Coffea arabica contienen una mayor concentración de lípidos y de sacarosa que C. canephora, mientras que en la composición de C. canephora hay mayor concentración de polisacáridos, cafeína, ácidos clorogénicos y cenizas. Dado que C. arabica confiere un sabor más fino y acentuado, se ha consolidado como la especie de mayor producción y valoración para el comercio de los granos (Kemsley et al., 1995; Sacco et al., 2011). Sin embargo, el sabor no solo depende de la especie, sino también del origen geográfico de las mismas y del procesamiento a que es sometido el grano, incluyendo el lavado vía húmeda, el cual permite obtener un balance entre acidez natural y aromas. Estos atributos son bien valorados en Estados Unidos y Alemania, los principales consumidores de café en el mundo (Puerta-Quintero, 2008; ICO, 2017).

Se cree que el uso del grano de café como bebida, tuvo comienzo con pueblos nativos de África, donde al igual que otras muchas plantas, se utilizaban las hojas, frutos y semillas del cafeto para el consumo humano. Sin embargo, el color rojo y la pulpa dulce (que contiene la cafeína estimulante) de los frutos, pudo haber atraído más su atención como fuente de alimento, y debido a su periodo corto de disponibilidad de la pulpa fresca, los llevó a masticar frutos secos, que con el paso del tiempo dio lugar al uso del café en mezclas sólidas con otros componentes (Teketay, 1999).

En la actualidad el café es una de las bebidas más populares y consumidas en el mundo, y es la segunda mercancía mayor comercializada después del petróleo (Mussatto et al., 2011; Nuhu, 2014). Se estima que ocupa el segundo lugar de consumo después del agua (Tofalo et al., 2016).

El grano de café contiene muchos compuestos bioactivos incluyendo cafeína, diterpenos, minerales, ácidos y ésteres, y compuestos fenólicos, muchos de los cuales muestran propiedades antioxidantes, anticancerígenas y antimicrobianas (Nuhu 2014; Cavalli y Tavani, 2016), que contribuyen a mejorar la salud en el consumidor (Santos y Lima, 2016). La especie, la madurez, la fermentación, el secado, el almacenamiento, el proceso de tostado y el método de preparación de la bebida influyen en la composición química y en la calidad del sabor, acidez, cuerpo y aroma de una taza de café (PuertaQuintero, 2008). Altos niveles de trigonelina y ácido 3,4-dicafeoilquínico se han relacionado con una buena calidad, y por el contrario, un mayor nivel de ácidos cafeoilquínicos y feruloilquínicos y sus productos de oxidación, a una mala calidad (Farah et al., 2006). Otras características químicas que favorecen la calidad del café son altos niveles de la actividad de la polifenol oxidasa, índice de color, azúcares y bajos niveles de acidez total, pH, conductividad eléctrica y lixiviación de potasio (Clemente et al., 2015). También se han utilizado las concentraciones de macros y micros elementos, para determinar la calidad de café y el 
origen geográfico del grano (Anderson y Smith, 2002), puesto que son dependientes de factores como el ambiente (temperatura, precipitación, altitud y latitud) (Neves et al., 2012), la especie, y la variedad (Leroy et al., 2006), el manejo agronómico (Temis-Pérez et al., 2011) y la composición química del suelo (Prieto et al., 2013; Clemente et al., 2015).

De acuerdo con Pohl et al. (2013), existe una variación en el contenido nutrimental de los granos de acuerdo al origen geográfico. De hecho, Oliveira et al. (2015) encontraron diferencias significativas entre los paises y entre los continentes en los perfiles minerales de muestras de granos de café. Partiendo de este preámbulo y dado que Brasil, México y Vietnam se encuentran entre los primeros 10 países productores de café a nivel mundial (Temis-Pérez et al., 2011; ICO, 2017), el presente trabajo tuvo como objetivo evaluar la variación nutrimental del grano de la variedad Robusta (Coffea canephora) de muestras provenientes de México, Brasil y Vietnam.

\section{MATERIALES Y MÉTODOS}

Muestras de granos de café de la variedad Robusta (Coffea canephora) de México, Brasil y Vietnam se secaron completamente, se trituraron, se pesaron y se sometieron a una digestión ácida en una mezcla de ácidos perclórico y nítrico, según el protocolo descrito por Alcántar y Sandoval (1999). Para determinar las concentraciones de macronutrimentos ( $\mathrm{P}, \mathrm{K}, \mathrm{Ca}, \mathrm{Mg}$ y $\mathrm{S})$ y micronutrimentos ( $\mathrm{B}, \mathrm{Cu}, \mathrm{Fe}, \mathrm{Mn}, \mathrm{Ni}$ y $\mathrm{Zn}$ ) en granos, se analizaron los extractos resultantes utilizando un espectrómetro de emisión atómica de inducción por plasma acoplado (ICP-AES) (Agilent ICP-AES, modelo 725-ES; Victoria, Australia). Las concentraciones de $\mathrm{N}$ se cuantificaron utilizando el método de semi-micro Kjeldahl de acuerdo al protocolo descrito por Bremner (1965).

Con los datos obtenidos se realizó un análisis de varianza para un diseño experimental completamente azar y cuando se detectaron diferencias estadísticas, se reali- zó la comparación de medias por el método de Tukey $(P \leq 0.05)$ con el paquete estadístico SAS.

\section{RESULTADOS Y DISCUSIÓN}

Las concentraciones de macronutrimentos en el grano de café de la variedad Robusta (Coffea canephora), de diferentes orígenes, son presentadas en el Cuadro 1. Con excepción del $\mathrm{N}$ que no presentó variación en la concentración entre los granos de diferentes orígenes, el resto de los macronutrimentos tuvieron concentraciones diferentes, observándose que los granos mexicanos tuvieron mayores valores, con excepción del K; y menores concentraciones se registraron en las muestras de Vietnam. Las variaciones entre las concentraciones altas y bajas encontradas, fueron mayores a $100 \%$ para $\mathrm{P}$ y Ca y superiores a $50 \%$ para $\mathrm{K}$ y $\mathrm{Mg}$, lo cual, corrobora que el origen de la muestra tiene una gran influencia en la concentración de elementos.

El comportamiento de la concentración de $\mathrm{N}$ es congruente con los resultados encontrados por Krivan y Morales (1993), quienes reportaron que no hay diferencias estadísticas en muestras de café verde de ocho países latinoamericanos. Además, las concentraciones reportadas por estos mismos autores, que fluctuaron entre 2.09 a $2.02 \%$, son cercanos a los obtenidos en el presente estudio. En un análisis de 60 muestras de café tostado expendidas en Polonia, Grembecka et al. (2007) reportaron un rango de concentración de 1776 a $2495 \mu \mathrm{g} \mathrm{g}^{-1} \mathrm{P}$, con una media de $2189 \pm 171 \mu \mathrm{g} \mathrm{g}^{-1} \mathrm{P}$, los cuales están por debajo de los valores obtenidos para la muestra proveniente de México; mientras que para K, reportan una variación de 9393 a $18793 \mu \mathrm{g} \mathrm{g}^{-1}$, valores por encimas de los concentraciones obtenidas para los tres países en estudio.

Con respecto al $\mathrm{Ca}$, las concentraciones, en muestras de café tostado tailandés, oscilan entre 529 a $781 \mu \mathrm{g} \mathrm{g}^{-1}$ (Ashu y Chandravanshi, 2011; Stelmanch et al., 2015), los cuales son inferiores a los resultados obtenidos en

\begin{tabular}{|c|c|c|c|c|c|c|}
\hline \multirow{2}{*}{$\begin{array}{l}\text { País de origen del } \\
\text { grano de café }\end{array}$} & \multirow{2}{*}{$\begin{array}{l}N \\
\%\end{array}$} & P & K & $\mathrm{Ca}$ & $\mathrm{Mg}$ & S \\
\hline & & \multicolumn{5}{|c|}{$\mu \mathrm{g} \mathrm{g}^{-1}$ de materia seca } \\
\hline México: & $2.8 \mathrm{a}$ & 7797.0 a & $3847.1 \mathrm{~b}$ & $2462.5 \mathrm{a}$ & 3564.2 a & $5328.7 \mathrm{a}$ \\
\hline Brasil: & $2.8 \mathrm{a}$ & $1677.4 \mathrm{~b}$ & $7278.0 \mathrm{a}$ & $1446.0 \mathrm{~b}$ & $2509.8 \mathrm{~b}$ & $1471.5 b$ \\
\hline Vietnam: & $2.8 \mathrm{a}$ & $1646.4 \mathrm{~b}$ & 7289.9 a & $1292.9 \mathrm{~b}$ & $2458.8 b$ & $1437.6 \mathrm{~b}$ \\
\hline
\end{tabular}

Medias con letra distinta en la misma columna son estadisticamente diferentes de acuerdo a la prueba de (Tukey, $P \leq 0.05)$. Cada valor es el promedio de tres repeticiones. 
muestras de los países en estudio. Sin embargo, con respecto a muestras de café expendido en el mercado polaco, cuyas concentraciones varían de 993 a $1505 \mu \mathrm{g}$ $\mathrm{g}^{-1} \mathrm{Ca}$ (Grembecka et al., 2007; Stelmach et al., 2013), las concentraciones de granos provenientes de Vietnam y Brasil entran dentro de este rango, no así para el de México, que estuvo por encima de estos valores.

Por otra parte, la concentración de Mg en los grano de café en estudio, estuvieron por debajo de los valores reportados para el café tostado de Etiopía $\left(1622 \mu \mathrm{g} \mathrm{g}^{-1}\right)$ y de Polonia (10745 y $1231 \mu^{g_{~ g}}{ }^{-1}$ ) (Ashu y Chandravanshi, 2011; Stelmanch et al., 2015).

Respecto al azufre, las muestras provenientes de México tuvieron las mayores concentraciones de este nutrimento (5328.7 $\mu \mathrm{g} \mathrm{g}^{-1}$ S) en comparación con las muestras provenientes de Brasil y Vietnam (1471.5 y $1437.6 \mu \mathrm{g} \mathrm{g}^{-1}$, respectivamente), entre las cuales no hubo diferencia estadísticas significativas. Sin embargo, en todos valores son muy inferiores a los reportados por Ballesteros et al. (2014), que en promedio fueron de $11700 \mu \mathrm{g} \mathrm{g}^{-1} \mathrm{~S}$ de café molido en base seca expendido en Portugal.

Las concentraciones de los macronutrimentos que encontramos en el presente estudio indican que el origen geográfico juega un papel importante la composición del grano, y por tanto influye en la calidad de la bebida. Krivan y Morales (1993) y Oliveira et al. (2015) han reportado variaciones nutrimentales en el grano y bebidas de café a nivel continental y mundial. Particularmente para los cafés mexicanos se ha demostrado que pueden diferenciarse geográficamente según su composición elemental, de tal forma que existe una gran variación nutrimental entre los granos producidos en el estado de Chiapas con respecto a los de Oaxaca (Muñiz-Valencia et al., 2014). Con respecto a Brasil, los factores químicos y ambientales son determinantes para discriminar la región de origen del café (Neves et al., 2012), lo cual concuerda con nuestros resultados.
Los micronutrimentos también presentaron variación en sus concentraciones de una especie a otra y de una región a otra (Cuadro 2). Sin embargo, solo fueron estadísticamente diferentes las de $\mathrm{Cu}, \mathrm{Fe}, \mathrm{Mn}$ y $\mathrm{Zn}$. Las muestras de grano de café mexicano, presentaron mayor concentración de Fe, Mn y Zn, superando en más de $100 \%$ a las de Brasil y Vietnam. Por el contrario, para $\mathrm{B}, \mathrm{Cu}$ y Ni las muestras de Brasil fueron superiores, no así las de Vietnam que fueron inferiores. Este comportamiento en la variación de la concentración de micronutrimentos en los granos de café, entre los países en estudio, son coincidentes con los reportados por Liu et al. (2014) y Anderson y Smith (2002), para 21 y 160 muestras de café respectivamente, procedente de diferentes paises de la región de África, América y Asia. Particularmente, para Fe, $\mathrm{Mn}$ y $\mathrm{Cu}$, se encontró factores de diferencias de 2.5, 2.0 y 1.5 respectivamente (Anderson y Smith, 2002).

En general, la concentración de los nutrimentos en el grano de café, presentan una variación dependiendo de la especie y la región de origen, por lo tanto, el genotipo y el origen geográfico de los cafés pueden ser determinantes del perfil químico de las bebidas (Anderson y Smith, 2002). Al respecto, Cruz et al. (2015) encontraron que el Mn es el mejor discriminador entre las áreas productoras de café de Colombia, Costa Rica, Cuba, El Salvador, México, Nicaragua, Panamá y Papúa Nueva Guinea, mientras que para las regiones de Indonesia, África Oriental y América Central y del Sur, podrían considerarse el $\mathrm{Cu}, \mathrm{Na}, \mathrm{Mn}$ y Fe. Aunque la concentración de elementos en el café es sólo alrededor del 5\%, parece ser un buen indicador de la autenticidad del grano y las mezclas (Pohl et al. (2013). Aparentemente, este indicador puede ofrecer información útil sobre patrones elementales individuales que son distintivos del origen de suelos en donde se desarrollan, además del cultivo y las condiciones ambientales utilizadas. Además, podrían indicar la calidad de la bebida y cantidad de minerales ingeridos. De acuerdo con Oliveira et al. (2015), el consumo regular de una taza de café expreso contribuye a

\begin{tabular}{|c|c|c|c|c|c|c|}
\hline \multirow{2}{*}{$\begin{array}{l}\text { País de origen del } \\
\text { grano de café }\end{array}$} & B & $\mathrm{Cu}$ & $\mathrm{Fe}$ & $M n$ & $\mathrm{Ni}$ & $\mathrm{Zn}$ \\
\hline & \multicolumn{6}{|c|}{$\mu \mathrm{g} \mathrm{g}^{-1}$ de materia seca } \\
\hline México & $9.9 \mathrm{a}$ & $15.8 \mathrm{~b}$ & 371.7 a & $84.2 \mathrm{a}$ & $1.5 \mathrm{a}$ & $194.4 \mathrm{a}$ \\
\hline Brasil & $10.2 \mathrm{a}$ & $18.8 \mathrm{a}$ & $42.9 \mathrm{~b}$ & $10.3 c$ & $1.8 \mathrm{a}$ & $5.7 b$ \\
\hline Vietman & $8.8 \mathrm{a}$ & $13.8 \mathrm{c}$ & $46.3 b$ & $18.6 \mathrm{~b}$ & nd & $4.1 \mathrm{~b}$ \\
\hline
\end{tabular}

Medias con letra distinta en la misma columna son estadisticamente diferentes de acuerdo a la prueba de (Tukey, $P \leq 0.05)$. Cada valor es el promedio de tres repeticiones. $n d=$ no detectado. 
una ingesta diaria de minerales que varía de $0.002 \%$ de $\mathrm{Na}$ en Centroamérica y hasta el $8.73 \% \mathrm{~K}$ en Asia.

La composición química, así como las características organolépticas del grano o la bebida de café, además de estar influenciadas por factores de tipo genético (Leroy et al., 2006) y agronómico (Temis-Pérez et al., 2011), también son modificadas por el clima (temperatura, precipitación) y el origen geográfico (altitud y latitud que son determinantes) (Neves et al., 2012). Este cultivo es muy sensible a las heladas, y la latitud es de suma importancia, no solo por el cambio de temperatura, sino también por los cambios en la radiación solar, dado que un aumento de los rayos UV puede degradar algunas fitohormonas, lo que a su vez modifica las características de las frutas (Toledo et al., 2016).

En el aspecto ambiental, la composición química del suelo y la relación de los elementos en éste son factores que también afectan la calidad y composición del grano de café. De hecho, la variación de la concentración nutrimental de los suelos de una región a otra y su interacción con el cultivo juegan un papel fundamental en la composición química del café (Rosas et al., 2008; Suárez et al., 2015). Se sabe que suelos ácidos con alto contenido de Fe y Al generan tazas de calidad intermedia y alta, mientras que tazas de calidad baja están asociadas con suelos moderadamente ácidos, con altos niveles de Cu, Zn, S y con bajos de Ca y Mg (Suárez et al., 2015). También existe una relación negativa entre la acidez en taza con el contenido de Ca y una correlación positiva entre la fragancia/ aroma con los contenidos de K, Na y Al en el suelo. De hecho, de acuerdo con Rosas et al. (2008) el Ca, Mg y Fe influyen en la fragancia; mientras que, el $N$ participa en el sabor, y el Cu actúa de forma negativa en la acidez.

En términos de manejo nutrimental, la aplicación de dosis óptimas de $K$ favorece el rendimiento, contenido de cafeína, fenoles totales y azúcares reducidos, pero también reduce la acidez total titulable, hasta un punto tal que puede afectar negativamente la calidad de la bebida (Clemente et al., 2015). Por otra parte, el Zn favorece la calidad de los granos, reduciendo el porcentaje de granos medianos y pequeños, la incidencia de daños por insectos, la lixiviación de $\mathrm{K}$ y conductividad eléctrica, entre otras, e incrementa el ácido clorogénico y la capacidad antioxidante (Prieto et al., 2013).

Las diferencias en la composición elemental de los cafés de distinto origen son considerables. Comprender esta variabilidad podría permitir manipular esos factores para producir una infusión de café más nutritiva y agradable, además de las ventajas que determinan la autenticidad del origen y la trazabilidad.

\section{CONCLUSIÓN}

E xiste una variación en la concentración de elementos minerales en los granos de café de la variedad Robusta de muestras provenientes de México, Brasil y Vietnam, donde los granos de café mexicano presentaron de manera general los valores de concentración nutrimental más altos. Los porcentajes de variación entre las concentraciones altas y bajas encontradas, para los macronutrimentos, fluctuaron en más de $100 \%$ para P y Ca y en más de 50\% para K y Mg. Las muestras de grano de café mexicano son las que presentaron mayor concentración de Fe, Mn y Zn, superando en más de 100\% a las provenientes de Brasil y Vietnam. Esto confirma que existe una variación en la concentración de elementos de acuerdo al origen geográfico de la muestra.

\section{LITERATURA CITADA}

Alcántar-González G., Sandoval-Villa M. 1999. Manual de análisis químico de tejido vegetal. Publicación Especial 10. Sociedad Mexicana de la Ciencia del Suelo. Chapingo, Estado de México. $156 \mathrm{p}$.

Anderson K.A., Smith B.W. 2002. Chemical profiling to differentiate geographic growing origins of coffee. J. Agric. Food Chem. 50: 2068-2075.

Ashu R., Chandravanshi B.S. 2011. Concentration levels of metals in commercially available ethiopian roasted coffee powders and their infusions. Bull. Chem. Soc. Ethiopia 25: 11-24.

Ballesteros L.F., Teixeira J.A., Mussatto S.I. 2014. Chemical, functional, and structural properties of spent coffee grounds and coffee silverskin. Food Bioprocess. Technol. 7: 3493-3503. doi: 10.1007/s11947-014-1349-z.

Bremner J.M. 1965. Total nitrogen. In: Black C.A. (ed.). Methods of soil analysis. Part 2. Agronomy 9. American Society of Agronomy. Madison, WI, USA. pp. 1149-1178.

Cavalli L., Tavani A. 2016. Coffee consumption and its impact on health. In: Wilson T., Temple N. (eds). Beverage Impacts on Health and Nutrition, Nutrition and Health. Springer, Switzerland. pp. 2947.

Clemente J.M., Prieto M.H.E., Alvares L.C., Finger F.L., Cecon P.R. 2015. Effects of nitrogen and potassium on the chemical composition of coffee beans and on beverage quality. Acta Sci. Agron. 37: 297-305

Cruz R., Morais S., Casal S. 2015. Mineral composition variability of coffees: A result of processing and production. In: Preddy $V$. (ed). Processing and Impact on Active Components in Food. Elsevier, The Netherlands. pp. 549-558.

Farah A., Monteiro M., Calado V., Franca A.S., Trugo L.C. 2006 Correlation between cup quality and chemical attributes of Brazilian coffee. Food Chem. 98: 373-380. 
Grembecka M. Malinowska E., Szefer P. 2007. Differentiation of market and its infusions in view of their mineral composition. Sci. Environ. 383: 59-69

ICO 2017. Seminars and Workshops. International Coffee Organization. http://www.ico.org/workshop.asp

Kemsley E.K., Ruault S., Wilson R.H. 1995. Discrimination between Coffea arabica and Coffea canephora variant robusta beans using infrared spectroscopy. Food Chem. 54: 321-326.

Krivan V., Barth P., Morales A.F. 1993. Multielement analysis of green coffee and its possible use for the determination of origin Mikrochim. Acta 110: 217-236.

Leroy T., Ribeyre F., Bertrand B., Charmentant P., Dufour M., Montagnon C., Marraccini P., Pot D. 2006. Genetics of coffee quality Brazilian J. Plant Physiol. 18: 229-242.

Liu H.C., You C.F., Chen C.Y., Liu Y.C., Chung M.T. 2014. Geographic determination of coffee beans using multi-element analysis and isotope ratios of boron and strontium. Food Chem. 142 439-445

Muñiz-Valencia R., Jurado J.M., Ceballos-Magaña S.G., Alcázar A., Hernández- Díaz J. 2014. Characterization of Mexican coffee according to mineral contents by means of multilayer perceptrons artificial neural networks. J. Food Compos. Anal. 34: 7-11.

Mussatto S.I., Machado E.M.S., Martins S., Teixeira J.A. 2011. Production, Composition and application of coffee and its industrial residues. Food Bioprocess Technol. 4: 661-672.

Neves B.J., Borem F.M., Cirillo M.A., Ribeiro M.M., Alves A.A. Ramos A.H.M. 2012. Coffee quality and its interactions with environmental factors in Minas Gerais, Brazilian J. Agric. Sci. 4: $181-190$

Nuhu A.A. 2014. Bioactive micronutrients in coffee: Recent analytical approaches for characterization and quantification. ISRN Nutrition. Article No. 384230

Oliveira M., Ramos S., Delerue-Matos C., Morais S. 2015. Espresso beverages of pure origin coffee: Mineral Characterization, contribution for mineral intake and geographical discrimination. Food Chem. 177: 330-338.

Pohl P., Stelmach E., Welma M., Szymezycha-Madeja A. 2013 Determination of the elemental composition of coffee using instrumental methods. Food Anal. Methods 6: 598-613.
Prieto M.H.E., Poltronieri Y Farah A., Perrone D. 2013. Zinc supplementation, production and quality of coffee beans. Rev. Ceres 60: 293-299

Puerta-Quintero G.I. 2008. Riesgos para la calidad por la comercialización de café pergamino húmedo. Avances Técnicos Cenicafé 373: 1-4.

Rosas A.J., Escamilla P.E., Ruiz R.O. 2008. Relación de los nutrimentos del suelo con las características Físicas y sensorales del café orgánico. Terra Latin. 26: 375-384

Sacco D.A.F., Belik W., Velleda N.C. 2011. The coffee planting in Brazil. Evolution, current situation and new challenges facing the future. Memoria Académica. DOI: http://www.memoria.fahce. unlp.edu.ar/art_revistas/pr.5036/pr.5036.pdf

Santos R.M.M., Lima D.R.A. 2016. Coffee health effects from early fetal development through childhood and adolescence. In: Hughes C.L., Waters M.D. (eds). Translational Toxicology, Molecular and Integrative Toxicology. Springer, Switzerland. pp. 321-337.

Stelmach E., Pohl P., Szymczycha-Madeja A. 2013. The suitability of the simplified method of the analysis of coffee infusions on the content of $\mathrm{Ca}, \mathrm{Cu}, \mathrm{Fe}, \mathrm{Mg}, \mathrm{Mn}$ and $\mathrm{Zn}$ and the study of the effect of preparation conditions on the leachability of elements into the coffee brew. Food Chem. 142: 1956-1961.

Stelmach E., Pohl P., Szymczycha-Madeja A. 2015. The content of Ca, $\mathrm{Cu}, \mathrm{Fe}, \mathrm{Mg}$ and $\mathrm{Mn}$ and antioxidant activity of green coffee brews. Food Chem. 182: 302-308.

Suárez S.J.C., Rodríguez B.E.H., Duran B.E.H. 2015. Efecto de als condicones de cultivo, las caractriticas químicas del suelo y el manejo de grano en los atributos sensoriales de café (Coffea arabica L.) en taza. Acta Agron. 64: 342-348.

Teketay D. 1999. History, botany and ecological requirements of coffee. Walia 20: 28-50.

Temis-Pérez A.L., López-Malo A., Sosa-Morales M.E. 2011. Producción de café (Coffea arabica L.): cultivo, beneficio, plagas y enfermedades. Tem. Sel. Ingen. Alim. 5: 54-74.

Tofalo R., Renda G., De Caterina R., Suzzi G. 2016. Coffee: Health Effects. In: Caballero B., Finglas P., Toldrá F. (eds.). Encyclopedia of Food and Health. Oxford, UK. pp. 237-243.

Toledo P.R.A.B., Pezza L., Pezza H.R., Toci A.T. 2016. Relationship between the different aspects related coffee quality and their volatile compounds. Compr. Rev. Food Sci. Food Saf. 15: 705719

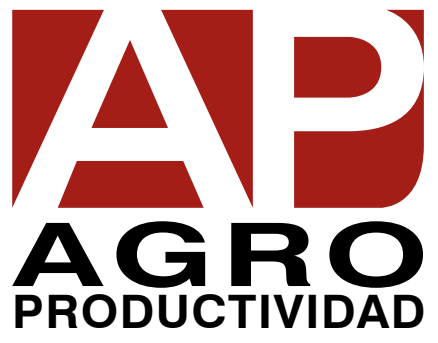

